ITP-UU-03/24

SPIN-2003/14

\title{
Sum over topologies and double-scaling limit in 2D Lorentzian quantum gravity
}

\author{
R. Loll* and W. Westra ${ }^{\dagger}$ \\ Institute for Theoretical Physics, Utrecht University, \\ Leuvenlaan 4, NL-3584 CE Utrecht
}

October 31, 2018

\begin{abstract}
We construct a combined non-perturbative path integral over geometries and topologies for two-dimensional Lorentzian quantum gravity. The Lorentzian structure is used in an essential way to exclude geometries with unacceptably large causality violations. The remaining sum can be performed analytically and possesses a unique and welldefined double-scaling limit, a property which has eluded similar models of Euclidean quantum gravity in the past.
\end{abstract}

\section{$1 \quad$ Summing over topologies?}

A central question that arises in the construction of a theory of quantum gravity is that of the fundamental, microscopic degrees of freedom whose dynamics the theory should describe. The idea that the information contained in the metric field tensor $g_{\mu \nu}$ may not constitute an adequate description of the geometric properties of spacetime at the very shortest scales goes back all the way to Riemann himself [1]. More

*email: r.loll@phys.uu.nl

†email: w.westra@phys.uu.nl 
recently this has led to the suggestion that at the Planck scale also the topological degrees of freedom of space-time should become excited. Wheeler is usually credited with coining the notion of a space-time foam [2], according to which space-time is a smooth, classical object macroscopically, well described by general relativity, but at the Planck scale presents a scenario of wildly fluctuating geometry and topology.

In the context of the gravitational path integral, this has inspired an extension of the customary integral over all metrics (modulo diffeomorphisms) by an additional sum over space-time topologies, namely,

$$
Z(\kappa, \lambda)=\sum_{\text {topol. }} \int D\left[g_{\mu \nu}\right] \mathrm{e}^{i S\left[g_{\mu \nu}\right]},
$$

where the square brackets denote diffeomorphism equivalence classes of metrics and where $S$ is the gravitational action. We will take the action to include a cosmological term,

$$
S=\int d^{d} x \sqrt{|\operatorname{det} g|}(\kappa R-\lambda),
$$

with $\kappa$ and $\lambda$ denoting the inverse gravitational coupling constant and the cosmological constant. In space-time dimension $d=4$, given the well-known difficulties of defining a path integral over the metric degrees of freedom alone, it may not come as a surprise that very little progress has been made in giving a well-defined mathematical and physical meaning to (11). Previous semi-classical treatments of topology change, as for example in discussions of the effect of baby universes on effective coupling constants (see 3] for a critical appraisal) are unlikely to be of relevance to the problem, for a variety of reasons.

First, since there is currently no direct or indirect evidence for topology changes from experiment, the phenomenon - if realized at all - must take place at the Planck scale or not too far from it, casting doubt on the applicability of semi-classical methods. Secondly, with very few exceptions, such investigations have been made within the path integral for Euclidean metrics. However, in the absence of a Wick rotation for theories with a dynamical metric, the Euclidean theory has no obvious relation with the physical, Lorentzian theory. Moreover, the Euclidean path integral seems to suffer from incurable divergences due to the presence of the conformal mode 4]. Lastly, and most importantly, once topology change is permitted, topology-changing contributions dominate the path integral completely, since the number of distinct geometries at a fixed space-time volume $V$ grows super-exponentially with $V$. This entropic effect is truly non-perturbative and cannot be seen in a semi-classical treatment. It implies that arguments for a dynamical suppression of topology changes which are based on an evaluation of their semi-classical action are largely irrelevant.

Yet more worrying for the proponents of a "sum over topologies" should be the fact 
that the analogous problem is unsolved even in dimension $d<4 .{ }^{1}$ Again, this can be traced to the super-exponential growth of the number of geometries with their volume, which renders the path integral badly divergent.

In space-time dimension two, which we will focus on in the following, the quantization of pure Euclidean (or Liouville) gravity for fixed topology is well understood in analytic terms [5]. The sum over topologies is turned into a sum over a single parameter $g \geq 0$, the genus (number of handles or holes) of the two-dimensional space-time. The Euclidean analogue of the path integral (11) for $d=2$ has been the object of intense study in the past, since it is an example of a non-perturbative sum over world sheets of a bosonic string (in a zero-dimensional target space) [6]. The problem has been addressed by matrix model methods or, equivalently, a regularization of the path integral in terms of triangulated, piecewise flat two-surfaces. However, it turns out that the topological expansion of (11) in powers of $\mathrm{e}^{-\kappa}$ (the integrated curvature in $2 \mathrm{~d}$ is proportional to $g$, up to an additive constant) is not Borel-summable, because the coefficients in the series grow factorially with $g$ and are all positive. Attempts to fix the ensuing non-perturbative ambiguities of the partition function in a unique and physically motivated way have so far remained unsuccessful [5].

\section{Doing it the Lorentzian way}

This leaves us in the rather unsatisfactory situation of not having a single instance of a quantum-gravitational theory where the sum over topologies had actually been performed. In the present work, we will suggest a possible way out of this impasse. The central idea is to take seriously the causal nature of space-time, and to perform a non-perturbative summation over Lorentzian geometries. As regards the sum over topologies, the Lorentzian structure will be used to quantify how badly causality is violated by individual contributions to the path integral. We will introduce and solve a model of $2 \mathrm{~d}$ quantum gravity which at the regularized level amounts to a sum over $2 \mathrm{~d}$ piecewise linear space-times of any genus whose causal properties are "not too bad". For the purposes of this paper, we will adopt a strictly quantum-gravitational point of view, in the sense that we will only be interested in models that do not lead to large-scale causality violations. In particular, we do not think that in this context third-quantized models, whose Hilbert spaces describe multiply-connected spatial geometries, can be interpreted in a physically meaningful way. This is different from situations where the geometries appear as imbedded quantities, as they do in the case of string theory,

\footnotetext{
${ }^{1}$ Quantum gravities in dimension 2 and 3 serve as useful models for diffeomorphism-invariant theories of dynamical geometry. Their metric configuration spaces and dynamics are much simplified in comparison with the physical, four-dimensional theory.
} 


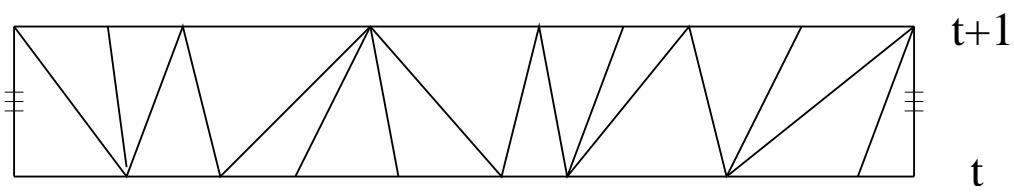

Figure 1: A strip $[t, t+1]$ of a $2 \mathrm{~d}$ Lorentzian triangulated space-time. The ends of the strip should be identified as indicated, leading to a compact spatial geometry $S^{1}$.

where moreover topology changes of the world sheet are mandatory, and not optional.

The question is then whether there are any models with topology change that produce a quantum space-time foam whose non-trivial microstructure leads to a measurable, but not necessarily large effect at a macroscopic level. The quantum gravity model we are about to construct has exactly this property. Although it is a model whose topological fluctuations are associated with the "mildest" type of causality violation imaginable in two dimensions, ${ }^{2}$ it is already at the limit of what is acceptable as a space-time foam. Namely, we will show that for a sufficiently large value of the renormalized gravitational coupling constant, the effects of topology change become overwhelming and the system enters a phase of "handle condensation".

In order to perform the sum (II) non-perturbatively, we will adopt a Lorentzian version of the regularized sum over piecewise flat $2 \mathrm{~d}$ space-times. For fixed topology $[0,1] \times S^{1}$, this model is exactly soluble and leads to a $2 \mathrm{~d}$ quantum gravity theory inequivalent to Liouville quantum gravity $[\underline{8}$, with a well-defined Wick rotation and without a $c=1$ barrier 9 . The difference can be traced to the absence (in the Lorentzian case) of branching "baby universes", which are incompatible with causality. ${ }^{3}$ This method of "Lorentzian dynamical triangulations" has also been applied successfully in dimension three [10], leading to a well-behaved quantum ground state, which unlike in the Euclidean theory does not degenerate into a lower-dimensional polymer as a consequence of a dominance of the conformal mode.

Recall that any $1+1$ dimensional Lorentzian space-time contributing to the regularized path integral is given by a sequence of strips of height $\Delta t=1$, where each strip in turn is a random sequence of $N$ Minkowskian up- and down-triangles (Fig】), each with two time-like and one space-like edge of length-squared $\pm a^{2}$ [8]. We will now generalize these to a class of Lorentzian geometries with holes, where the holes have minimal time duration $\Delta t=1$. Although this time interval goes to zero in the continuum limit $a \rightarrow 0$, their effect is not necessarily negligible, since the triangle density goes to infinity in the limit. The way in which we create triangulations with holes in

\footnotetext{
${ }^{2}$ However, it should be kept in mind that all topology changes in 2 and $3 \mathrm{~d}$ which do not involve universe creation or annihilation are "bad" according to the classification of Dowker and collaborators [7].

${ }^{3}$ By this we mean baby universes that do not return to the "mother universe", and therefore do not change the space-time topology. We will not consider such configurations in the present work.
} 


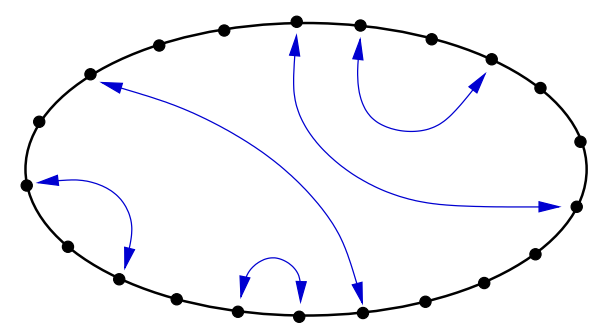

Figure 2: Horizontal section through a strip $[t, t+1]$ of triangles, with time-like edges appearing as dots. The arrows indicate where the circle should be glued and cut to obtain (in this case six) disconnected cylinder components.

a strip $[t, t+1]$ is as follows. Suppose the geometry has been built up to integer time $t$, possibly with holes. The spatial geometry at time $t$ is a closed circle consisting of $l_{t}$ space-like edges. Now, glue on another strip with matching "in-geometry" of length $l_{t}$ and some "out-geometry" of length $l_{t+1}$ (giving rise to a total discrete strip volume of $N_{t}=l_{t}+l_{t+1}$ triangles). Next, glue an even number of the $N_{t}$ time-like edges in the strip pairwise to each other, according to an arrow diagram (Fig 2), and then cut open the geometry at each of these edges, perpendicular to the direction in which they were glued together. This will result in a space-time geometry consisting of several cylindrical components between $t$ and $t+1$. In order to obtain back a spatial circle at time $t+1$, the cylinders must be cut open at some of their vertices at $t+1$ and their spatial boundaries be aligned in some order to form again a single $S^{1}$. In this way, one has constructed a strip geometry with some number $g_{t}$ of holes.

It is straightforward to show that if arbitrary regluings at time $t+1$ are allowed, the number of possible geometries at a given strip volume scales factorially with $N_{t}$, just as in the Euclidean case. However, if one looks at the causal properties of the resulting space-times, most of them turn out to be extremely ill-behaved, in the sense that even a single hole in the entire space-time will lead to a global rearrangement of parts of a light front after passing the hole, even if it exists only for an infinitesimal time $\Delta t$. Fortunately, there is a subclass of geometries for which this does not happen, which are those where the cylinders are reglued without any intermediate relative twisting or rearrangement of the order of the components during the time interval when they are disconnected.

The effect is most easily illustrated by the case of two components. In a "regluing without twist" the two saddle points ${ }^{4} p_{t}$ and $p_{t+1}$ at $t$ and $t+1$ where the hole appears and vanishes are connected in each of the two cylinders by a time-like link, which implies that they are nearest lattice neighbours in either of the components. If this

\footnotetext{
${ }^{4}$ Note in passing that it is not clear a priori how to account for the curvature singularities at the saddle points in the Lorentzian action, and how to treat them in the Wick rotation, see [1] for a related discussion. We will simply use the standard Regge prescription in terms of deficit angles in the Wick-rotated action.
} 
is not the case, e.g. if one of the cylinders is twisted before regluing, then $p_{t}$ and $p_{t+1}$ will not appear as nearest neighbours in that component, but $p_{t+1}$ will have a relative shift $\Delta l$ along the spatial direction. The resulting space-time geometry will have the property that a light beam of macroscopic width that passes by the hole will be split into two parts which will emerge with a relative separation of $\Delta l$ after the hole disappears! By comparison, the only effect of the hole in the "untwisted" case is that a small fraction of the light beam will be scattered into the far-away part of the space-time to which the hole connects during its infinitesimal life time (this effect is of course also present in the twisted case). ${ }^{5}$ Since we find it difficult to envisage how a quantum geometry with anything near a macroscopic causal structure could emerge from a superposition of such ill-behaved manifolds, our sum over topologies will contain only geometries with "untwisted" holes.

\section{Discrete solution and double-scaling limit}

To illustrate that the causality constraints imposed above do lead to a well-defined and soluble model, we will now solve the combinatorics for a single strip $\Delta t=1$ and look for a scaling behaviour of the two coupling constants that leads to a non-trivial continuum limit. The partition function after Wick-rotating is

$$
Z(\lambda, \kappa)=\sum_{l_{\text {in }}} \sum_{l_{\text {out }}} \mathrm{e}^{-\lambda\left(l_{\text {in }}+l_{\text {out }}\right)} \sum_{\left.T\right|_{l_{\text {in }}, l_{\text {out }}}} \mathrm{e}^{-\kappa g(T)},
$$

with a sum over the initial and final boundary geometries of length $l_{\text {in }}$ and $l_{\text {out }}$, and a sum over triangulations $T$ of a strip with these boundaries. For a given triangulated strip of volume $N=l_{\text {in }}+l_{\text {out }}$, the counting of geometries with holes according to the procedure of the previous section involves a counting of diagrams like in Fig 2 with $N$ vertices and $g$ arrows. Expression (3) can be rearranged,

$$
Z(\lambda, \kappa)=\frac{1}{2} \sum_{N=0}^{\infty} \sum_{g=0}^{[N / 2]}\left(\begin{array}{l}
N \\
2 g
\end{array}\right) \frac{(2 g) !}{g !(g+1) !} \mathrm{e}^{-2 \kappa g} \mathrm{e}^{-(\lambda-\log 2) N}
$$

after which the sums can be performed explicitly, leading to

$$
Z(\lambda, \kappa)=\frac{1}{2\left(1-\mathrm{e}^{-(\lambda-\log 2)}\right)} \frac{1-\sqrt{1-4 z}}{2 z},
$$

where the second term depends only on the combination

$$
z:=\mathrm{e}^{-2 \kappa}\left(\mathrm{e}^{\lambda-\log 2}-1\right)^{-2} .
$$

\footnotetext{
${ }^{5} \mathrm{~A}$ detailed geometric analysis can be found in [12].
} 
An infinite-volume limit is obtained by tuning the bare cosmological coupling $\lambda$ to $\log 2$ from above ${ }^{6}$, as in standard Lorentzian quantum gravity [8],

$$
\lambda=\lambda^{c r i t}+a^{2} \Lambda+O\left(a^{3}\right) \equiv \log 2+a^{2} \Lambda+O\left(a^{3}\right),
$$

where $\Lambda$ denotes the renormalized, dimensionful cosmological constant, as the geodesic cutoff $a \rightarrow 0$. As can be seen from eq.(5), this is only consistent if simultaneously also the inverse Newton constant $\kappa$ is renormalized. Such a double-scaling limit is obtained by fixing $z$ to a constant, $z=c<1 / 4$, and defining a renormalized coupling $\mathrm{K}$ by

$$
\mathrm{K}=\kappa-2 \log \frac{1}{a \sqrt{\Lambda}}+O(a), \quad \mathrm{K}:=\frac{1}{2} \log \frac{1}{c} .
$$

Substituting these expansions into the expression for the strip partition function (5D), a straightforward computation yields the renormalized partition function in terms of $\Lambda$ and the gravitational coupling $G=1 / \mathrm{K}$,

$$
Z^{R}(\Lambda, G)=\frac{\mathrm{e}^{2 / G}}{4 \Lambda}\left(1-\sqrt{1-4 \mathrm{e}^{-2 / G}}\right)
$$

In the continuum theory, one expects $\Lambda$ to set the global scale because of $\langle V\rangle=\frac{1}{\Lambda}$ for the expectation value of the space-time volume. On the other hand, the strength of the gravitational coupling governs the average number $\langle g\rangle$ of holes per slice, which is proportional to the fraction of a lightbeam that will be scattered by holes in a non-local and causality-violating manner [12]. As is illustrated by Fig 3] for $G=0$ there are no holes at all. Their number increases for $G>0$, first slowly and then rapidly, until it diverges at the maximum value $G=2 / \log 4$, at which point the system undergoes a transition to a phase of "condensed handles".

What we have found therefore is an example of a gravity-inspired statistical model with a well-defined double-scaling limit. As in previous work on non-perturbative gravitational path integrals, the Lorentzian structure of the individual geometries has played a crucial role in the construction. In forthcoming work [13] we will investigate what happens when one keeps the boundaries of the space-time strip fixed instead of summing over them, as presented here. This more complicated model needs to be solved in order to determine the Hamiltonian and the full propagator of 2D Lorentzian quantum gravity with holes. Interestingly, it turns out that the inclusion of the boundaries leads to a different scaling behaviour of Newton's constant. This also implies a different behaviour for the number of holes: unlike in the strip model, there is no condensation of handles, and the number of holes per strip stays infinitesimal. Un-

\footnotetext{
${ }^{6}$ Note that this gives rise to a non-negative renormalized cosmological constant; our approach naturally leads to a de-Sitter-like behaviour.
} 


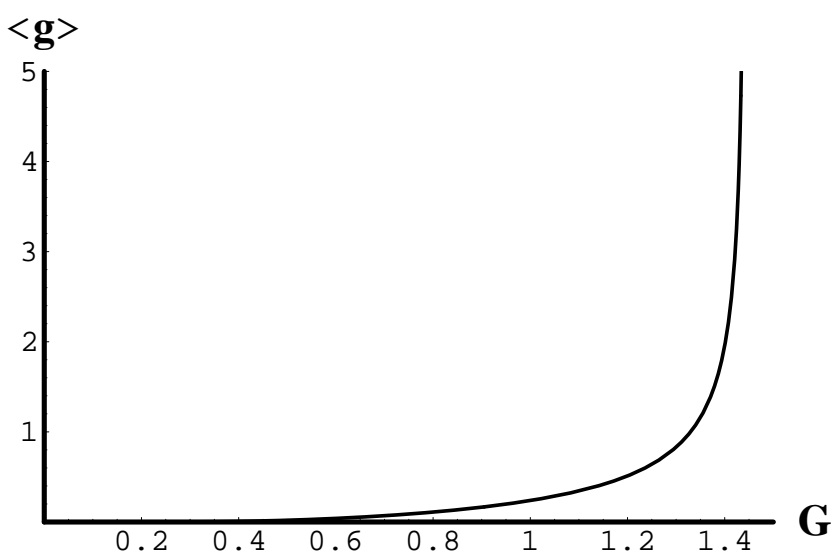

Figure 3: The average number $\langle g\rangle$ of holes as a function of Newton's constant $G$ in the one-strip model described by the partition function (91).

like in the pure Lorentzian theory without holes therefore, the scaling of the "bulk" couplings and the bulk partition function cannot be deduced from solving the simpler strip model with summed-over boundaries. This may teach us an important lesson for higher-dimensional models, where a similar phenomenon may well be present.

Acknowledgements. We thank J. Ambjørn, G. 't Hooft and S. Zohren for enjoyable discussions. Support through the EU network on "Discrete Random Geometry", grant HPRN-CT-1999-00161, is gratefully acknowledged.

\section{References}

[1] http://www.maths.tcd.ie/pub/HistMath/People/Riemann/Geom

[2] J.A. Wheeler, Ann. Phys. 2, 604 (1957).

[3] S.B. Giddings, Int. J. Mod. Phys. A5, 3811 (1990).

[4] A. Dasgupta and R. Loll, Nucl. Phys. B 606, 357 (2001) hep-th/0103186.

[5] F. David, in Gravitation and Quantizations, ed. J. Zinn-Justin and B. Julia, NorthHolland, 679 (1995) hep-th/9303127; P. Di Francesco, P. Ginsparg and J. ZinnJustin, Phys. Rept. 254, 1 (1995); J. Ambjørn, B. Durhuus and T. Jonsson, Quantum Geometry, Cambridge Monogr. Math. Phys. 1 (1997).

[6] M.R. Douglas and S.H. Shenker, Nucl. Phys. B 335, 635 (1990); E. Brézin and V.A. Kazakov, Phys. Lett. B 236, 144 (1990); D.J. Gross and A.A. Migdal, Phys. Rev. Lett. 64, 127 (1990). 
[7] F. Dowker, in The future of theoretical physics and cosmology, ed. G.W. Gibbons, E.P.S. Shellard and S.J. Rankin, Cambridge Univ. Press, 436 (2003) gr-qc/0206020.

[8] J. Ambjørn and R. Loll, Nucl. Phys. B 536, 407 (1998) hep-th/9805108.

[9] J. Ambjørn, K.N. Anagnostopoulos and R. Loll, Phys. Rev. D 61, 044010 (2000) hep-lat/9909129.

[10] J. Ambjørn, J. Jurkiewicz and R. Loll, Phys. Rev. D 64, 044011 (2001) hep-th/0011276.

[11] J. Louko and R.D. Sorkin, Class. Quant. Grav. 14, 179 (1997) gr-qc/9511023.

[12] R. Loll and W. Westra, Acta Phys. Polonica B 34, 4997 (2003) hep-th/0309012.

[13] R. Loll, W. Westra and S. Zohren (to be published). 